\title{
Erythrocyte count is associated with prognosis in Chinese patients with primary biliary cholangitis
}

\author{
YINGHAO CHANG $^{1,2}$, CHANGCUN GUO ${ }^{1}$, GUANYA GUO ${ }^{1}$, ZHOU YUAN $^{1}$, XINMIN ZHOU ${ }^{1}$, \\ JINGBO WANG $^{1}$, ZHEYI HAN ${ }^{1}$, YU CHEN $^{1}$, GUI JIA ${ }^{1}$ and YING HAN ${ }^{1}$ \\ ${ }^{1}$ Department of Digestive Diseases, Xijing Hospital, Fourth Military Medical University, Xi'an, Shaanxi 710032; \\ ${ }^{2}$ Department of Gastroenterology and Hepatology, General Hospital of Western Theater Command, \\ Chengdu, Sichuan 610083, P.R. China
}

Received March 23, 2019; Accepted November 11, 2019

DOI: $10.3892 /$ etm.2020.8446

\begin{abstract}
Erythroid parameters have been indicated to be important prognostic factors for liver diseases. The present study aimed to evaluate the prognostic value of the erythrocyte count in Chinese patients with primary biliary cholangitis (PBC) and develop a prognostic model. The clinical data of 301 patients with PBC were retrospectively reviewed. Univariate and multivariate Cox regression analysis was performed to identify potential prognostic risk factors. Bivariate correlation analysis was used to determine the correlation coefficient of the erythrocyte count and biochemical indices. The prognostic values of different factors were compared by receiver operating characteristic (ROC) curve analysis. A novel prognostic model was constructed using multivariate logistic regression. Multivariate regression analysis suggested that the erythrocyte count was an independent risk factor/prognostic index $(\mathrm{P}=0.042)$. The erythrocyte count in peripheral blood decreased as the histological stage progressed $(\mathrm{P}<0.001)$. The erythrocyte count was correlated with albumin, liver stiffness and Fibrosis-4. Compared with that of platelets, the area under the ROC curve of the erythrocyte
\end{abstract}

Correspondence to: Professor Ying Han, Department of Digestive Diseases, Xijing Hospital, Fourth Military Medical University, 127 Changle West Road, Xi'an, Shaanxi 710032, P.R. China

E-mail: hanying1@fmmu.edu.cn

Abbreviations: $\mathrm{PBC}$, primary biliary cholangitis; UDCA, ursodeoxycholic acid; RDW, red blood cell distribution width; ALP, alkaline phosphatase; ULN, upper limit of normal; GGT, gamma-glutamyltranspeptidase; HGB, haemoglobin; HCT, hematocrit; MCV, erythrocyte mean corpuscular volume; PLT, platelet; ALT, alanine aminotransferase; AST, aspartate aminotransferase; ALB, albumin; GLB, globulin; TBIL, total bilirubin; CHO, cholesterol; TG, triglyceride; CRE, creatinine; PT, prothrombin time; APTT, activated partial thromboplastin time; TT, thrombin time; LS, liver stiffness; FIB-4, fibrosis-4

Key words: erythrocyte count, primary biliary cholangitis, prognosis, predictive model, risk factor count was significantly greater. A similar area under the ROC curve was determined for the erythrocyte count, albumin and total bilirubin $(\mathrm{P}>0.05)$. A novel prognostic model was established as follows: $\mathrm{P}=1 /\{1+\mathrm{e}-[6.140-3.193 \times \mathrm{Ln}$ (erythrocyte count) $-0.184 \mathrm{x}$ albumin $+0.827 \mathrm{x} \operatorname{Ln}$ (total bilirubin)]\}. The novel model had a comparable prognostic value to that of the GLOBE score and UK-PBC risk score, and had a better performance than the Mayo risk score at baseline ( 0.838 vs. 0.787). In conclusion, the erythrocyte count is an independent risk factor/prognostic index in Chinese patients with PBC. It was correlated with liver function and fibrosis in Chinese patients. The novel model incorporating the erythrocyte count and biochemical indices at baseline may serve as a prognostic tool in Chinese patients with PBC (Trial registration number, ChiCTR-ONRC-10002070; date of registration, 2010-05-10).

\section{Introduction}

Primary biliary cholangitis (PBC) is a chronic and progressive immune-mediated cholestatic liver disease (1). The survival periods of patients with PBC vary. For patients diagnosed at the early stage, the survival periods are similar to those of healthy individuals after ursodeoxycholic acid (UDCA) treatment (2). The median survival time is 6-10 years for patients with advanced PBC (3). According to clinical practice guidelines, risk stratification has a vital role in the clinical management of patients with PBC (4). For risk stratification of patients with $\mathrm{PBC}$, it is required to assess the probability of adverse events and perform a prediction of the prognosis prior to treatment. In order to predict the survival of patients with PBC, several prognostic models based on clinical parameters have been developed, including the GLOBE score, UK-PBC risk score and Mayo risk score (5-8).

All of the continuous scoring systems mentioned above have been widely used for prognostic evaluation in patients with PBC. Furthermore, several studies were performed to explore novel prognostic indices or models $(5,7)$. Their results suggested that existing prognostic models of PBC may be improved by taking other variables into account. In previous years, erythroid-associated parameters have been indicated to be linked to liver-associated diseases $(9,10)$. A previous study has reported that the erythrocyte count in peripheral blood 
was associated with survival after surgery in patients with primary liver cancer (11). Several other studies have reported that the red blood cell distribution width (RDW) may serve as a predictive index for histological severity and a potential prognostic indicator of chronic liver diseases (12-14). While the mechanism underlying the clinical relevance of erythrocytes in PBC remains elusive, previous studies have indicated that erythroid parameters, including the erythrocyte count, are potential prognostic indicators for PBC $(12,13)$.

In the present study, the prognostic value of the erythrocyte count in Chinese patients with PBC was analyzed. Correlations of the erythrocyte count with liver-associated indices were investigated. Furthermore, a novel predictive model for the prognosis of PBC was developed by incorporating the erythrocyte count and other biochemical indices.

\section{Patients and methods}

Patients. The present study was approved by the Ethics Committee of Xijing Hospital (Xi'an, China) and all patients had signed an informed consent form. The present study was performed in accordance with the Declaration of Helsinki. The present study retrospectively enrolled 301 patients with $\mathrm{PBC}$ who received treatment at the Department of Gastroenterology at Xijing Hospital (Xi'an, China) from March 2006 to August 2018. Initiation of UDCA treatment was between March 2006 and January 2017. The inclusion criteria were as follows: i) Patients diagnosed with PBC meeting at least two of the following criteria: Alkaline phosphatase (ALP) $>2$-fold of the upper limit normal (ULN) or gamma-glutamyl transpeptidase (GGT) $>5$-fold of the ULN, titer of anti-mitochondrial antibody $>1: 40$ and liver biopsy exhibiting florid bile duct lesions; ii) UDCA treatment was initiated and maintained for at least 12 months at the dosage of $13-15 \mathrm{mg} / \mathrm{kg} /$ day after diagnosis of PBC. The exclusion criteria were as follows: i) Concurrence of other liver diseases, including viral hepatitis, primary sclerosing cholangitis, autoimmune hepatitis, alcoholic liver disease, hemochromatosis, Wilson's disease or non-alcoholic steatohepatitis; ii) baseline clinical data were incomplete; iii) for patients with transplant-free survival, the duration of follow-up was $<1$ year. All patients had been followed up at intervals of 1-6 months during UDCA treatment. The ULN of ALP was $150 \mathrm{U} / 1$ and the ULN of GGT was $50 \mathrm{U} / 1$.

Study design. Clinical data were retrieved from the patients records at baseline and after 1 year of treatment. The data included the erythrocyte count, haemoglobin (HGB), hematocrit (HCT), erythrocyte mean corpuscular volume (MCV), age, sex, platelets (PLT), alanine aminotransferase (ALT), aspartate aminotransferase (AST), albumin (ALB), globulin (GLB), total bilirubin (TBIL), ALP, GGT, cholesterol, triglyceride (TG), creatinine (CRE), prothrombin time (PT), activated partial thromboplastin time (APTT), fibrinogen and thrombin time. Other demographic indices, including body mass index and status of smoking and drinking, were not included in the analysis, as they were not available. The GLOBE score and UK-PBC risk score were calculated after 1 year of treatment and the Mayo risk score was determined at baseline. Liver stiffness (LS) (15) and Fibrosis-4 (FIB-4) (16) were recorded at baseline and taken as indicators of liver fibrosis. LS was detected by transient elastography with FibroTouch (Wuxi Hisky Medical Technology Co., Ltd.). According to the formula in the Formulae of predictive models section below, the FIB-4 score was determined from four baseline variables, including age, AST, ALT and PLT. Pathological stages were classified according to Ludwig's classification (17) and presented as categorical data. The end-points were defined as liver transplantation or death.

Histological analysis. Liver biopsies were analyzed by two experienced hepatic pathologists (Professor Zengshan Li and Dr Lin Chen, Department of Pathology, Xijing Hospital, Fourth Military Medical University). Histological features were recorded as stage 1 (portal stage), stage 2 (periportal stage), stage 3 (septal stage) and stage 4 (cirrhosis) $(17,18)$, according to Ludwig's classification.

Statistical analysis. Continuous variables with a normal distribution are expressed as the mean \pm standard deviation, while variables with a non-normal distribution are presented as the median (lower quartile-upper quartile). Shapiro-Wilk test was applied to assess normality of distribution. Categorical variables were expressed as n (\%). For comparison of categorical data, Fisher's exact test was performed. The Kruskal-Wallis $\mathrm{H}$-test and Mann-Whitney U-test with Bonferroni correction were used for continuous values with a non-normal distribution. Hazard ratios (HRs) and 95\% CIs of biochemical parameters were calculated using the Cox regression model in univariate and multivariate analyses. Bivariate correlation analysis was applied to determine correlations between erythrocyte count and liver-associated parameters and Spearman correlation coefficients were obtained. Receiver operating characteristic (ROC) curve analysis was utilized for evaluating the prognostic value of biochemical parameters and predictive models. Comparisons of areas under receiver operating characteristic curve (AUCs) of biochemical parameters and predictive models were accomplished by the Delong-Delong non-parametric method. Based on binary multivariate logistic regression analysis, a predictive model was constructed for estimating the prognosis at baseline. The Hosmer-Lemeshow test was applied to test for the goodness of fit of the predictive model. Analyses were accomplished using SPSS 19.0 (IBM Corp.), except for the comparisons of AUCs, which were performed with MedCalc Statistical Software version 15.2.2 (MedCalc Software bvba). The P-values were two-sided and $\mathrm{P}<0.05$ was considered to indicate a statistically significant difference.

Formulae of predictive models. i) GLOBE score $=0.044378$ $\mathrm{x}$ age (years) at baseline $+0.93982 \mathrm{x} \ln (\mathrm{TBIL}$ times ULN at one-year follow-up) $+0.335648 \times \ln$ (ALP times the ULN at one-year follow-up) $-2.266708 \times$ ALB times the lower limit of normal (LLN) at one-year follow-up -0.002581 x PLT count per $10^{9} / 1$ at one-year follow-up $+1.216865(6)$.

ii) UK-PBC risk score $=1-0.982^{0.0287854} \times$ (ALP at one-year follow-up/ULN-1.722136304) $-0.0422873 \times\{[1 /(\mathrm{AST}$ at one-year follow-up/ULN/10)] $-8.675729006\}+1.4199 x[\ln ($ TBIL at one-year follow-up/ULN/10) +2.709607778$]-1.960303 x$ (ALB/LLN-1.17673001) -0.4161954 x (PLT/LLN-1.873564875) (7).

iii) FIB-4=[age (years) x AST (U/1)]/[PLT $\left(10^{9} / 1\right) \times$ $\sqrt{\text { ALT (U/l) }}](16)$. 
Table I. Features at baseline and after one year of therapy.

\begin{tabular}{|c|c|c|c|c|}
\hline Parameter & Normal limit & Baseline & After one year of therapy & P-value \\
\hline Erythrocytes $\left(10^{12} / 1\right)$ & $>4.00$ & $3.94(3.52-4.29)$ & $4.24(3.88-4.56)$ & $<0.001$ \\
\hline $\operatorname{HGB}(\mathrm{g} / \mathrm{l})$ & $>120.00$ & $119.00(105.00-131.00)$ & $127.00(111.00-137.00)$ & $<0.001$ \\
\hline $\operatorname{HCT}(\%)$ & $>40.0$ & $36.7(32.5-39.5)$ & $38.9(34.8-41.5)$ & $<0.001$ \\
\hline MCV (fl) & $>80.00$ & $93.00(89.80-96.20)$ & $92.40(87.98-95.78)$ & 0.064 \\
\hline $\operatorname{PLT}\left(10^{9} / 1\right)$ & $>100.00$ & $137.00(83.00-202.00)$ & $146.00(84.50-210.00)$ & 0.304 \\
\hline ALT (U/l) & $<40.00$ & $58.00(37.00-94.00)$ & $33.00(23.00-55.75)$ & $<0.001$ \\
\hline $\operatorname{AST}(\mathrm{U} / \mathrm{l})$ & $<40.00$ & $63.00(43.00-96.00)$ & $38.00(29.00-59.75)$ & $<0.001$ \\
\hline ALB (g/l) & $>35.00$ & $39.20(36.00-41.80)$ & $43.00(39.90-45.50)$ & $<0.001$ \\
\hline GLB (g/l) & $<32.00$ & $30.75(28.00-34.40)$ & $31.80(28.50-35.10)$ & 0.178 \\
\hline TBIL $(\mu \mathrm{mol} / \mathrm{l})$ & $<17.10$ & $19.00(12.70-32.00)$ & $14.50(10.83-21.40)$ & $<0.001$ \\
\hline $\operatorname{ALP}(\mathrm{U} / \mathrm{l})$ & $<150.00$ & $263.00(161.00-427.50)$ & $162.50(111.00-243.25)$ & $<0.001$ \\
\hline GGT (U/l) & $<50.00$ & $268.00(118.50-425.00)$ & $101.50(42.00-211.50)$ & $<0.001$ \\
\hline $\mathrm{CHO}(\mathrm{mmol} / \mathrm{l})$ & $<5.18$ & $4.43(3.65-5.71)$ & $4.85(3.92-5.60)$ & 0.090 \\
\hline $\mathrm{TG}(\mathrm{mmol} / \mathrm{l})$ & $<1.70$ & $1.17(0.77-1.69)$ & $1.25(0.92-1.84)$ & 0.006 \\
\hline $\mathrm{CRE}(\mu \mathrm{mol} / \mathrm{l})$ & $<133.00$ & $75.00(66.00-84.00)$ & $80.00(72.00-88.50)$ & $<0.001$ \\
\hline PT $(\sec )$ & $<13.00$ & $12.90(12.20-13.90)$ & $12.90(12.30-13.70)$ & 0.929 \\
\hline APTT (sec) & $<37.00$ & $39.85(36.20-43.73)$ & $38.75(35.48-42.58)$ & 0.161 \\
\hline Fibrinogen $(\mathrm{g} / \mathrm{l})$ & $>2.00$ & $2.96(2.50-3.52)$ & $3.00(2.51-3.54)$ & 0.862 \\
\hline $\mathrm{TT}(\mathrm{sec})$ & $<16.00$ & $17.20(16.40-18.00)$ & $17.10(16.30-18.40)$ & 0.571 \\
\hline Age (years) & & $51.89 \pm 9.98$ & & \\
\hline Female sex $(\%)$ & & $258(85.7 \%)$ & & \\
\hline Follow-up time ${ }^{\mathrm{a}}$ (months) & & $44(27-60)$ & & \\
\hline Stage $(1 / 2 / 3 / 4)$ & & $22 / 119 / 48 / 65$ & & \\
\hline Transplant-free survival ${ }^{\mathrm{b}}(\%)$ & & $260(86.4 \%)$ & & \\
\hline $\mathrm{LS}(\mathrm{kPa})$ & & $9.91(6.82-19.56)$ & & \\
\hline FIB-4 & & $3.287(1.836-6.383)$ & & \\
\hline Mayo risk score & & $4.847(4.251-5.708)$ & & \\
\hline GLOBE score & & & $0.191(-0.468-1.053)$ & \\
\hline UK-PBC risk score & & & $0.0306(0.0153-0.0726)$ & \\
\hline
\end{tabular}

${ }^{\text {aD }}$ Duration from diagnosis to the end of follow-up or reaching the clinical end-point. ${ }^{\mathrm{b} S u r v i v a l}$ without liver-associated death or liver transplantation. Continuous data are presented as the mean \pm standard deviation or as the median with interquartile range and categorical data are expressed as the number with percentage. HGB, haemoglobin; HCT, hematocrit; MCV, erythrocyte mean corpuscular volume; PLT, platelets; ALT, alanine aminotransferase; AST, aspartate aminotransferase; ALB, albumin; GLB, globulin; TBIL, total bilirubin; ALP, alkaline phosphatase; GGT, gamma-glutamyltranspeptidase; CHO, cholesterol; TG, triglyceride; CRE, creatinine; PT, prothrombin time; APTT, activated partial thromboplastin time; TT, thrombin time; LS, liver stiffness; FIB-4, Fibrosis-4.

iv) Mayo risk score $=0.039 \times$ age (years) $+0.871 \times \ln [$ serum bilirubin $(\mathrm{mg} / \mathrm{dl})]-2.53 \times \ln [$ albumin $(\mathrm{mg} / \mathrm{dl})]+2.38 \mathrm{x}$ $\ln [$ prothrombin time $(\mathrm{sec})]+0.859 \mathrm{x}$ ascites; (ascites: no $=0$, yes=1) (8).

\section{Results}

Patient characteristics. The demographic and clinical data of the present cohort are provided in Table I. A total of 301 Chinese patients with $\mathrm{PBC}$ were retrospectively reviewed, including $258(85.7 \%)$ females and $43(14.3 \%)$ males. The average age of the patients at baseline was 51.89 years. The median follow-up time was 44 months. Furthermore, 41 patients $(13.6 \%)$ underwent liver transplantation or died during the follow-up.
Prognostic factors of survival by univariate and multivariate regression analysis. According to the univariate Cox regression analysis, the baseline erythrocyte count, MCV, HGB, HCT, age, PLT, AST, ALB, GLB, TBIL, CRE, PT and APTT were associated with patient survival. A multivariate analysis was then performed for each of these baseline factors. The results indicated that only the erythrocyte count $(\mathrm{P}=0.042$, HR: 0.524, 95\%CI: 0.281-0.976), ALB (P<0.001, HR: 0.844, 95\%CI: 0.792-0.900) and TBIL $(\mathrm{P}<0.001$, HR: $1.008,95 \% \mathrm{CI}$ : 1.005-1.012) at baseline were independent risk factors and associated with patient prognosis (Table II).

Correlation of erythrocyte count with biochemical and fibrosis indices. Bivariate correlation analysis was used for determining linear correlation coefficients between the 
Table II. Prognostic value of baseline parameters in Cox regression analysis.

\begin{tabular}{|c|c|c|c|c|c|c|}
\hline \multirow[b]{2}{*}{ Baseline parameter } & \multicolumn{3}{|c|}{ Univariate analysis } & \multicolumn{3}{|c|}{ Multivariate analysis } \\
\hline & $\mathrm{P}$-value & Hazard ratio & $95 \% \mathrm{CI}$ & P-value & Hazard ratio & $95 \% \mathrm{CI}$ \\
\hline Age & $<0.001$ & 1.059 & $1.021-1.099$ & & & \\
\hline Sex (female/male) & 0.960 & 0.978 & $0.411-2.328$ & & & \\
\hline Erythrocyte & $<0.001$ & 0.246 & $0.166-0.365$ & 0.042 & 0.524 & $0.281-0.976$ \\
\hline HGB & $<0.001$ & 0.969 & $0.958-0.981$ & & & \\
\hline $\mathrm{HCT}$ & $<0.001$ & 0.001 & $0.000-0.001$ & & & \\
\hline $\mathrm{MCV}$ & 0.304 & 1.008 & $0.993-1.022$ & & & \\
\hline PLT & 0.007 & 0.994 & 0.989-0.998 & & & \\
\hline ALT & 0.725 & 0.999 & $0.994-1.004$ & & & \\
\hline AST & 0.057 & 1.003 & $1.000-1.006$ & & & \\
\hline ALB & $<0.001$ & 0.819 & $0.782-0.858$ & $<0.001$ & 0.844 & $0.792-0.900$ \\
\hline GLB & 0.053 & 1.044 & 0.999-1.091 & & & \\
\hline TBIL & $<0.001$ & 1.011 & $1.008-1.014$ & $<0.001$ & 1.008 & $1.005-1.012$ \\
\hline ALP & 0.410 & 1.000 & $0.999-1.002$ & & & \\
\hline GGT & 0.341 & 0.999 & $0.998-1.001$ & & & \\
\hline $\mathrm{CHO}$ & 0.776 & 0.978 & $0.838-1.141$ & & & \\
\hline $\mathrm{TG}$ & 0.910 & 0.979 & $0.684-1.403$ & & & \\
\hline CRE & 0.028 & 0.973 & 0.949-0.997 & & & \\
\hline PT & $<0.001$ & 1.385 & $1.215-1.579$ & & & \\
\hline APTT & 0.001 & 1.076 & $1.030-1.124$ & & & \\
\hline Fibrinogen & 0.234 & 0.779 & $0.516-1.175$ & & & \\
\hline $\mathrm{TT}$ & 0.973 & 0.999 & $0.953-1.047$ & & & \\
\hline
\end{tabular}

HGB, haemoglobin; HCT, hematocrit; MCV, erythrocyte mean corpuscular volume; PLT, platelets; ALT, alanine aminotransferase; AST, aspartate aminotransferase; ALB, albumin; GLB, globulin; TBIL, total bilirubin; ALP, alkaline phosphatase; GGT, gamma-glutamyltranspeptidase; CHO, cholesterol; TG, triglyceride; CRE, creatinine; PT, prothrombin time; APTT, activated partial thromboplastin time; TT, thrombin time.

erythrocyte count and other baseline biochemical indices (Table SI). Close correlations were identified between the erythrocyte count and other parameters, including the PLT, ALB and TBIL $(\mathrm{P}<0.001)$. A positive correlation of the erythrocyte count with ALB $(r=0.555)$ and PLT $(r=0.329)$ was determined, as well as a negative correlation with TBIL $(r=-0.406)$. The correlations between the erythrocyte count and hepatic fibrosis parameters were investigated to verify whether the erythrocyte count in the peripheral blood was associated with the progression of PBC. The erythrocyte count decreased as the pathological stage increased $(\mathrm{P}<0.001$; Fig. 1; Table SII). There was a negative correlation between the erythrocyte count and the pathological stage $(r=-0.410$, $\mathrm{P}<0.001$; Fig. S1). Furthermore, a bivariate correlation analysis was performed, suggesting that the erythrocyte count was closely linearly correlated to $\mathrm{LS}(\mathrm{r}=-0.581, \mathrm{P}=0.003)$ and FIB-4 ( $\mathrm{r}=-0.482, \mathrm{P}<0.001)$, indicating that the erythrocyte count was associated with the progression of cirrhosis in Chinese patients with PBC (Fig. 2).

Predictive performance of erythroid parameters for patient prognosis. The prognostic value of various biochemical indices was determined using ROC curve analysis at baseline and at the one-year follow-up. At baseline, the erythrocyte count had a significantly higher AUC value than HGB (0.822 vs. 0.762 ,

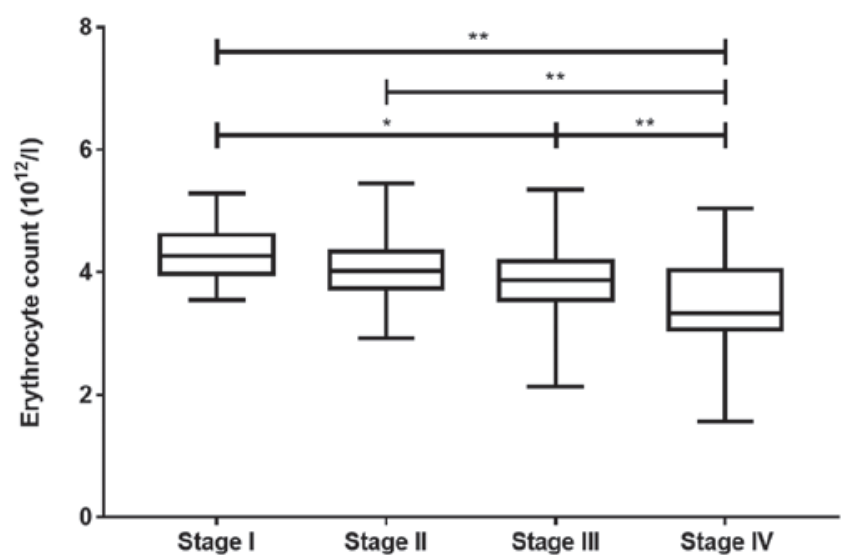

Figure 1. Comparison of erythrocyte count between patients with different histological stages. ${ }^{*} \mathrm{P}<0.05,{ }^{* *} \mathrm{P}<0.01$.

$\mathrm{P}<0.001)$, HCT (0.822 vs. 0.782, $\mathrm{P}=0.002)$ and PLT (0.822 vs. 0.654, $\mathrm{P}=0.003$; Fig. 3A). After UDCA treatment for one year, the erythrocyte count still had a significantly higher AUC value than PLT (0.821 vs. 0.676, $\mathrm{P}=0.006$; Fig. 3B; Table III). The AUC of the erythrocyte count was comparable to that of ALB and TBIL $(\mathrm{P}>0.05)$ at baseline and at the one-year follow-up (Table SIII). 
Table III. Prognostic value of parameters at baseline and after one year of therapy.

A, Baseline

\begin{tabular}{|c|c|c|c|c|}
\hline \multirow[b]{2}{*}{ Parameter } & \multirow[b]{2}{*}{ Area } & \multirow[b]{2}{*}{ P-value } & \multicolumn{2}{|c|}{$\begin{array}{l}\text { Asymptotic } 95 \% \\
\text { confidence interval }\end{array}$} \\
\hline & & & Lower & Upper \\
\hline Erythrocyte count & 0.822 & $<0.001$ & 0.738 & 0.906 \\
\hline HGB & 0.762 & $<0.001$ & 0.674 & 0.851 \\
\hline HCT & 0.782 & $<0.001$ & 0.693 & 0.870 \\
\hline PLT & 0.654 & 0.002 & 0.563 & 0.744 \\
\hline ALB & 0.849 & $<0.001$ & 0.785 & 0.914 \\
\hline TBIL & 0.765 & $<0.001$ & 0.687 & 0.843 \\
\hline
\end{tabular}

$\mathrm{B}$, After one year of treatment

Asymptotic $95 \%$

confidence interval

\begin{tabular}{|c|c|c|c|c|}
\hline \multirow[b]{2}{*}{ Parameter } & \multirow[b]{2}{*}{ Area } & \multirow[b]{2}{*}{ P-value } & \\
\hline & & & Lower & Upper \\
\hline Erythrocyte count & 0.821 & $<0.001$ & 0.730 & 0.912 \\
\hline HGB & 0.783 & $<0.001$ & 0.687 & 0.879 \\
\hline HCT & 0.807 & $<0.001$ & 0.715 & 0.898 \\
\hline PLT & 0.676 & 0.003 & 0.575 & 0.777 \\
\hline ALB & 0.835 & $<0.001$ & 0.743 & 0.927 \\
\hline TBIL & 0.846 & $<0.001$ & 0.772 & 0.921 \\
\hline
\end{tabular}

HGB, haemoglobin; HCT, hematocrit; PLT, platelets; ALB, albumin; TBIL, total bilirubin.
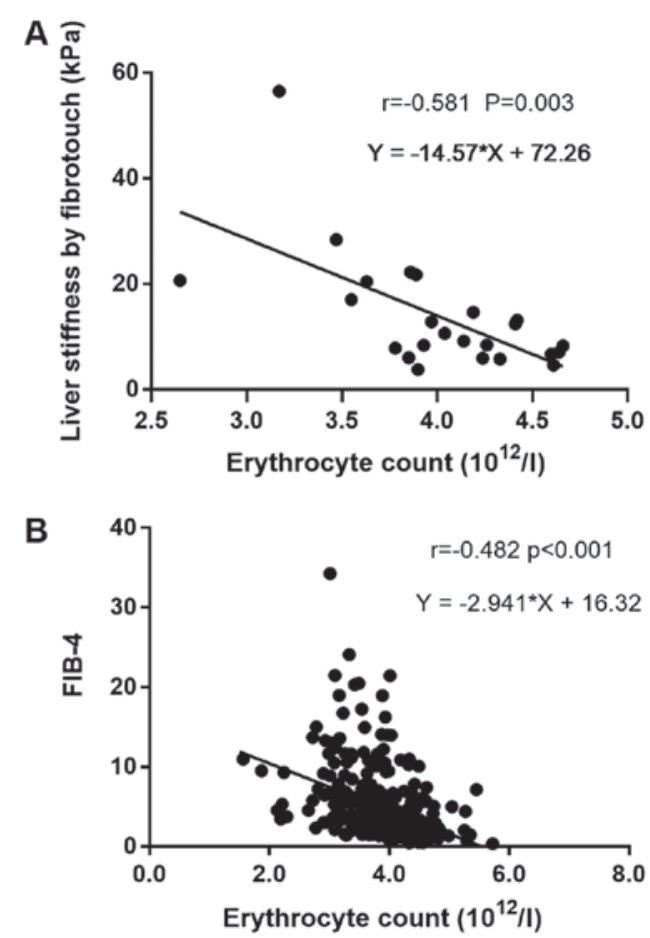

Figure 2. Correlation between erythrocyte count and fibrosis indexes in scatter plots. (A) Correlation between erythrocyte count and liver stiffness. (B) Correlation between erythrocyte count and FIB-4. FIB-4, Fibrosis-4.
Derivation of predictive model for prognosis. Multivariate logistic regression analysis was performed on potential prognostic risk factors to construct a predictive model. Only variables identified in the multivariate Cox regression were included in the model. Ln transformations were applied for erythrocyte count and TBIL. The predictive model was constructed as follows:

$$
\mathrm{P}=1 /\left\{1+\mathrm{e}^{-[6.140-3.193 \times \text { Ln(erythrocyte count) }-0.184 \times \mathrm{ALB}+0.827 \times \text { LnTBIL }]}\right\} .
$$

Regarding goodness of fit, the result of the HosmerLemeshow test was $\mathrm{P}=0.454$. Subsequently, the performance of the novel predictive model was compared using the GLOBE score, UK-PBC risk score and Mayo risk score. At baseline, the novel model had a greater AUC value than the Mayo risk score $(0.838$ vs. 0.787$)$, while the novel model had a similar AUC value compared with the GLOBE score and the UK-PBC risk score ( $\mathrm{P}>0.05$; Fig. 4; Table IV; Table SIV). The sensitivity and specificity of the predictive model were 80.0 and $85.1 \%$ for predicting transplant-free survival according to the maximum Youden index.

\section{Discussion}

Abnormalities of peripheral blood erythroid parameters have been observed in several liver diseases. In recent years, certain 
Table IV. Receiver operating characteristic parameters presenting the prognostic value of predictive score systems.

\begin{tabular}{lcccccc}
\hline & \multicolumn{5}{c}{$\begin{array}{c}\text { Asymptotic } \\
95 \% \text { confidence } \\
\text { interval }\end{array}$} \\
\cline { 4 - 6 } Scoring system & Area under curve & P-value & Lower & Upper & Sensitivity $(\%)$ & Specificity (\%) \\
\cline { 4 - 6 } Predictive model $(>0.16)$ & 0.838 & $<0.001$ & 0.747 & 0.929 & 80.0 & 85.1 \\
Mayo risk score $(>6.10)$ & 0.787 & $<0.001$ & 0.668 & 0.907 & 74.4 & 88.5 \\
GLOBE score $(>0.83)$ & 0.893 & $<0.001$ & 0.812 & 0.974 & 92.9 & 76.4 \\
UK-PBC risk score $(>0.092)$ & 0.884 & $<0.001$ & 0.809 & 0.959 & 78.6 & 87.2 \\
\hline
\end{tabular}

A

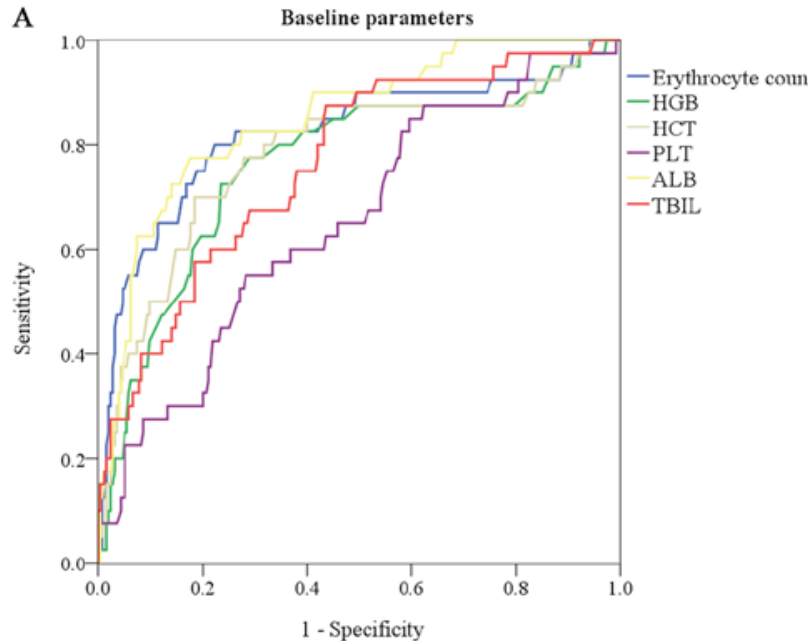

B

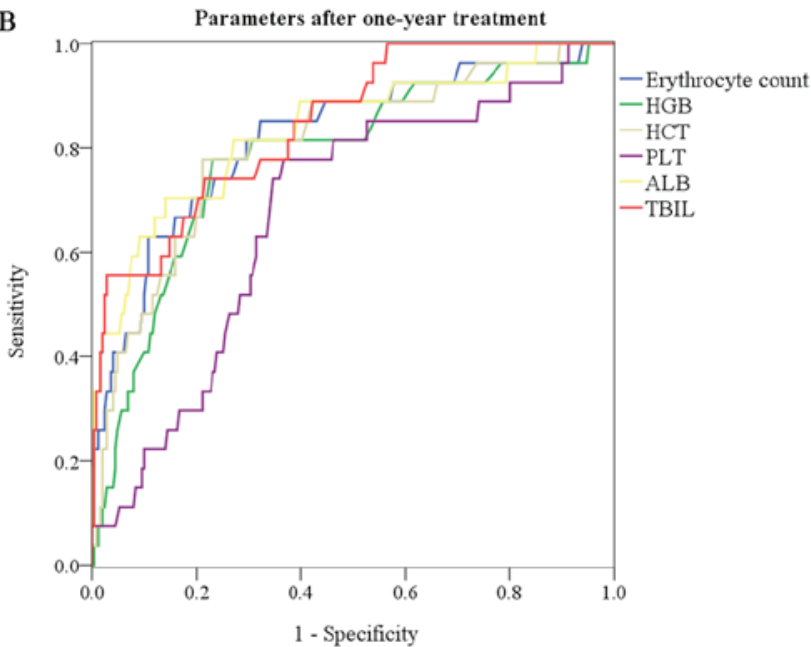

Figure 3. Receiver operating characteristic curves presenting the prognostic value of parameters at baseline and after one year of therapy. (A) Prognostic value of various baseline parameters. (B) Prognostic value of parameters after one year of therapy. HGB, haemoglobin; HCT, hematocrit; PLT, platelet; ALB, albumin; TBIL, total bilirubin.

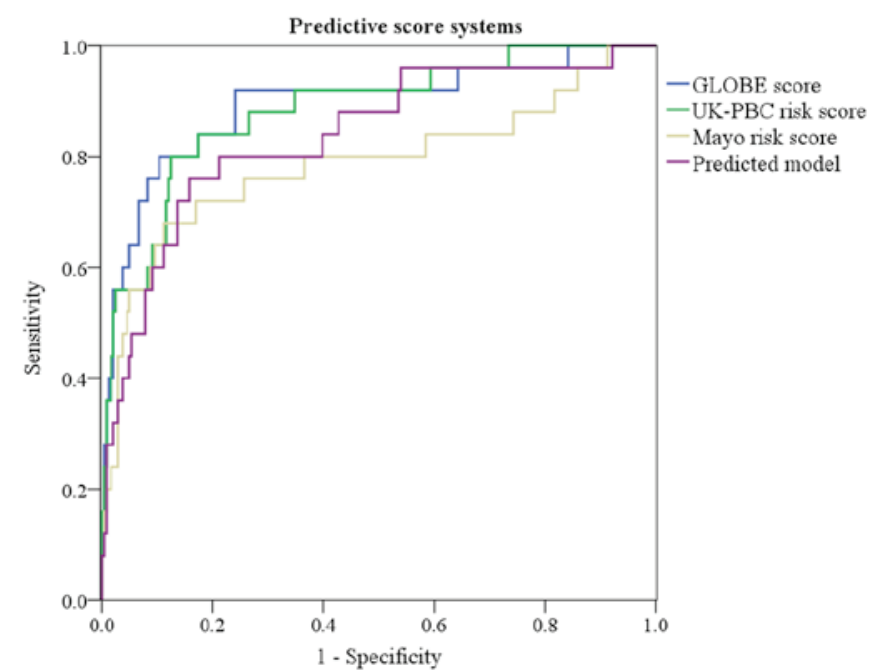

Figure 4. Receiver operating characteristic curves presenting the prognostic value of various predictive score systems.

studies with limited patients indicated a potential prognostic value of erythroid parameters in patients with PBC $(12,13)$. The decrease in erythrocyte count may be attributed to several aspects. As a common reason for the decrease of the erythocyte count, hemolysis presents in up to $50 \%$ of patients with chronic liver disease regardless of etiologies (19). Complications of cirrhosis, including splenomegaly, are the major reasons for hemolysis in patients with end-stage disease. Alterations of the lipid composition, which were detected in the erythrocyte membrane of patients with liver diseases, have been associated with liver damage $(20,21)$. The erythrocyte count in peripheral blood may be associated with chronic hepatic injury due to the impairment of the erythrocyte membrane structure caused by alteration of the lipid composition. Furthermore, certain studies suggested that the deficiency of hematopoietic precursors due to immune injury of the hematopoietic system was also linked to a reduced erythrocyte count in patients with PBC $(22,23)$. Other causes of erythrocyte deficiency included malnutrition, iron deficiency, bone marrow depression, cirrhosis and medications $(22,24)$.

Splenomegaly is a cause of decreased erythrocyte count in patients with end-stage PBC and it is considered to be linked to poor prognosis. However, in the present study, splenomegaly was not subjected to multivariate Cox regression analysis, as the results of the imaging examination at baseline were not available for all patients. The relevance of splenomegaly regarding the prognostic value of the erythrocyte 
count requires to be clarified. The mechanisms underlying the correlations between erythrocytes and liver fibrosis or dysfunction in patients with $\mathrm{PBC}$ also require to be further clarified. In the present study, a close correlation between erythrocyte count and liver function parameters, including ALB and TBIL, was identified. Furthermore, the erythrocyte count decreased as the pathological stage and liver fibrosis progressed. The increase in erythrocyte count after UDCA treatment was accompanied by the improvement of liver function and liver fibrosis score. Therefore, the decrease of the erythrocyte count may reflect the severity of liver disease and the extent of liver function deficiency in patients with PBC, which is in accordance with a previous study in patients with primary liver cancer (11).

Early prognostic models are important for risk stratification for patients with PBC. In the present study, the prognostic value of the erythrocyte count was investigated as a simple, routinely tested parameter in Chinese patients with PBC. The novel predictive model incorporating the erythrocyte count had a better prognostic performance than the Mayo risk score, one of the most widely used prognostic systems (25), and was comparable with the GLOBE score and UK-PBC risk score in the evaluation of the prognosis regarding transplant-free survival. The GLOBE score and UK-PBC risk score involve parameters after one year of UDCA treatment. The novel model included only parameters at baseline, which may be helpful for early prognosis evaluation prior to initiation of UDCA treatment.

The present study has certain limitations. First, the present study was a retrospective study performed in only one clinical center. More prospective studies are required to confirm the results of the present study. Furthermore, no validation of the novel model was performed in another PBC cohort. The present cohort was only comprised of Chinese patients with PBC from one hospital. Although the sample size of the cohort was relatively big, it is still required to validate the prognostic model in other cohorts in China and other populations. Furthermore, RDW has been reported to be of prognostic value in PBC patients. RDW data were missing from the clinical data of the present cohort, making it impossible to verify the results of previous studies and to determine whether RDW is a surrogate parameter for the erythrocyte count in the prognosis of PBC.

In conclusion, the present study indicated that the erythrocyte count is a useful prognostic parameter in Chinese patients with PBC. The peripheral blood erythrocyte count was closely correlated with liver dysfunction and fibrosis. The model incorporating the erythrocyte count, ALB and TBIL may serve as an early prognostic tool for PBC.

\section{Acknowledgements}

The authors would like to thank Professor Zengshan Li and Dr Lin Chen (Department of Pathology, Xijing Hospital, Fourth Military Medical University) for the analyses of liver biopsies.

\section{Funding}

The present study was funded by the National Natural Science Foundation of China (grant no. 81770569).

\section{Availability of data and materials}

The datasets used and/or analyzed during the present study are available from the corresponding author on reasonable request.

\section{Authors' contributions}

YiC conceived experiments, recruited patients, and acquired and analyzed data. CG contributed to the conception of the experiment and revised the manuscript. GG, ZY and GJ contributed to the acquisition of data and the patient recruitment. $\mathrm{XZ}, \mathrm{JW}, \mathrm{ZH}, \mathrm{YuC}$ reviewed the manuscript and contributed to the interpretation of results. YH designed experiments and reviewed the manuscript.

\section{Ethics approval and consent to participate}

This study was approved by the Ethics Committee of Xijing Hospital (Xi'an, China) and all patients had provided written informed consent. The study was performed in accordance with the Declaration of Helsinki.

\section{Patient consent for publication}

Not applicable.

\section{Competing interests}

The authors declare that they have no competing interests.

\section{References}

1. Hirschfield GM and Gershwin ME: The immunobiology and pathophysiology of primary biliary cirrhosis. Ann Rev Pathol 8: 303-330, 2013.

2. Parés A, Caballería L and Rodés J: Excellent long-term survival in patients with primary biliary cirrhosis and biochemical response to ursodeoxycholic Acid. Gastroenterol 130: 715-720, 2006.

3. Carey EJ, Ali AH and Lindor KD: Primary biliary cirrhosis. Lancet 386: 1565-1575, 2015.

4. European Association for the Study of the Liver. Electronic address: easloffice@easloffice.eu; European Association for the Study of the Liver: EASL clinical practice guidelines: The diagnosis and management of patients with primary biliary cholangitis. J Hepatol 67: 145-172, 2017.

5. Chen S, Duan W, You H and Jia J: A brief review on prognostic models of primary biliary cholangitis. Hepatol Int 11: 412-418, 2017.

6. Lammers WJ, Hirschfield GM, Corpechot C, Nevens F, Lindor KD, Janssen HL, Floreani A, Ponsioen CY, Mayo MJ, Invernizzi P, et al: Development and validation of a scoring system to predict outcomes of patients with primary biliary cirrhosis receiving ursodeoxycholic acid therapy. Gastroenterology 149: 1804-1812, 2015.

7. Carbone M, Sharp SJ, Flack S, Paximadas D, Spiess K, Adgey C, Griffiths L, Lim R, Trembling P, Williamson K, et al: The UK-PBC riskscores: Derivationand validation of ascoring systemforlong-term prediction of end-stage liver disease in primary biliary cholangitis. Hepatology 63: 930-950, 2016.

8. Lammers WJ,Kowdley KV and van Buuren HR: Predicting outcome in primary biliary cirrhosis. Ann Hepatol 13: 316-326, 2014.

9. Hu Z, Sun Y, Wang Q, Han Z, Huang Y, Liu X, Ding C, Hu C, Qin Q and Deng A: Red blood cell distribution width is a potential prognostic index for liver disease. Clin Chem Lab Med 51: 1403-1408, 2013.

10. Wei TT, Tang QQ, Qin BD, Ma N, Wang LL, Zhou Lin and Zhong RQ: Elevated red blood cell distribution width is associated with liver function tests in patients with primary hepatocellular carcinoma. Clin Hemorheol Microcirc 64: 149-155, 2016.

11. Xie X, Yao M, Chen X, Lu W, Lv Q, Wang K, Zhang L and Lu F: Reduced red blood cell count predicts poor survival after surgery in patients with primary liver cancer. Medicine 94: e577, 2015. 
12. Wang LL, Wei TT, Yin JR, Qin BD, Ma N, Tang QQ, Zhou L and Zhong RQ: Red blood cell distribution width and mean platelet volume are potential prognostic indices for patients with primary biliary cirrhosis. Clin Chem Lab Med 55: e127-e129, 2017.

13. Wang H, Xu H, Wang X, Wu R, Gao X, Jin Q and Niu J: Red blood cell distribution width to platelet ratio is related to histologic severity of primary biliary cirrhosis. Medicine (Baltimore) 95 e3114, 2016.

14. Wang H, Xu H, Qu L, Wang X, Wu R, Gao X, Jin Q and Niu J: Red blood cell distribution width and globulin, noninvasive indicators of fibrosis and inflammation in chronic hepatitis patients. Eur J Gastroenterol Hepatol 28: 997-1002, 2016.

15. Roccarina D, Rosselli M, Genesca J and Tsochatzis EA Elastography methods for the non-invasive assessment of portal hypertension. Expert Rev Gastroenterol Hepatol 12: 155-164, 2018.

16. Tsai E and Lee TP: Diagnosis and evaluation of nonalcoholic fatty liver disease/nonalcoholic steatohepatitis, including noninvasive biomarkers and transient elastography. Clin Liver Dis 22 73-92, 2018.

17. Chan AW, Chan RC, Wong GL, Wong VW, Choi PC, Chan HL and To KF: Evaluation of histological staging systems for primary biliary cirrhosis: Correlation with clinical and biochemical factors and significance of pathological parameters in prognostication. Histopathology 65: 174-186, 2014.

18. Reshetnyak VI: Primary biliary cirrhosis: Clinical and laboratory criteria for its diagnosis. World J Gastroenterol 21: 7683-7708, 2015.

19. Gonzalez-Moreno EI, Martinez-Cabriales SA, Cruz-Moreno MA Borjas-Almaguer OD, Cortez-Hernandez CA, Bosques-PadillaFJ, Garza AA, Gonzalez-Gonzalez JA, Garcia-Compean D, Ocampo-Candiani J and Maldonado-Garza HJ: Primary biliary cholangitis associated with warm autoimmune hemolytic anemia. J Dig Dis 17: 128-131, 2016.
20. Arendt BM, Ma DW, Simons B, Noureldin SA, Therapondos G, Guindi M, Sherman M and Allard JP: Nonalcoholic fatty liver disease is associated with lower hepatic and erythrocyte ratios of phosphatidylcholine to phosphatidylethanolamine. Appl Physiol Nutr Metab 38: 334-340, 2013.

21. Notarnicola M, Caruso MG, Tutino V, Bonfiglio C, Cozzolongo R, Giannuzzi V, De Nunzio V, De Leonardis G, Abbrescia DI, Franco I, et al: Significant decrease of saturation index in erythrocytes membrane from subjects with non-alcoholic fatty liver disease (NAFLD). Lipids Health Dis 16: 160, 2017.

22. Tsikrikoni A, Rigopoulou EI, Zachou K, Liaskos C, Kyriakou D and Dalekos GN: Bone marrow findings in patients with autoimmune liver diseases. J Gastroenterol Hepatol 23: e416-e421, 2008.

23. Kyriakou DS, Alexandrakis MG, Zachou K, Passam F, Stathakis NE and Dalekos GN: Hemopoietic progenitor cells and bone marrow stromal cells in patients with autoimmune hepatitis type 1 and primary biliary cirrhosis. J Hepatol 39: 679-685, 2003.

24. Karagöz E and Tanoglu A: Red blood cell distribution width: A potential prognostic index for liver disease? Clin Chem Lab Med 52: e201, 2014.

25. Purohit T and Cappell MS: Primary biliary cirrhosis: Pathophysiology, clinical presentation and therapy. World J Hepatol 7: 926-941, 2015. 